\title{
Hymenoscyphus fraxineus and two new Hymenoscyphus species identified in Korea
}

\author{
A. Gross • J. G. Han
}

Received: 17 November 2014 / Revised: 24 February 2015 / Accepted: 26 February 2015 / Published online: 25 March 2015

(C) German Mycological Society and Springer-Verlag Berlin Heidelberg 2015

\begin{abstract}
Hymenoscyphus fraxineus is an invasive fungal pathogen that causes ash dieback in Europe. Recent investigations have identified $H$. fraxineus on herbarium specimens in Korea. In this paper, these specimens, plus five additional collections, were studied by internal transcribed spacer (ITS) screening and subsequent phylogenetic analysis using three additional sequence markers (actin, calmodulin, EF1- $\alpha$ ). Using the concept of genealogical concordance phylogenetic species recognition (GCPSR), H. fraxineus was confirmed in five of the collections on petioles of Fraxinus mandshurica and $F$. chinensis subsp. rhynchophylla. The remaining collections revealed two novel species, both occurring on petioles of F. chinensis subsp. rhynchophylla. They are described as Hymenoscyphus occultus sp. nov. and Hymenoscyphus koreanus sp. nov., based on morphological and molecular data. Both develop a Chalara-like anamorph similar to that of H. fraxineus. Together with the newly described H. albidoides from China and H. linearis from Japan, the clade containing H. fraxineus now consists of six species. Within this clade, H. koreanus forms a sister species to H. albidus and both share highly similar morphological and molecular features. Hymenoscyphus occultus is more distantly related to
\end{abstract}

Electronic supplementary material The online version of this article (doi:10.1007/s11557-015-1035-1) contains supplementary material, which is available to authorized users.

\section{A. Gross $(\bowtie)$}

ETH Zurich, Department of Environmental Systems Science, Forest Pathology and Dendrology, Institute of Integrative Biology (IBZ), ETH Zurich, 8092 Zurich, Switzerland

e-mail: andrin.gross@usys.ethz.ch

\section{J. G. Han}

Mushroom Research Division, Rural Development Administration, National Institute of Horticultural and Herbal Science,

Eumseong 369-873, Korea
$H$. fraxineus and shows proximity to $H$. linearis. Ascocarp production on ash leaf malt-extract agar could be shown for the two new species, and for H. linearis and $H$. albidus. The experiment demonstrated these species' ability to self-fertilize. Our findings suggest the diversity of Hymenoscyphus species on Fraxinus sp. might be higher than currently known, calling for further investigations on petioles of other Fraxinus species.

Keywords Chalara fraxinea $\cdot$ Hymenoscyphus pseudoalbidus $\cdot$ Molecular phylogeny · Fraxinus platypoda . Hymenoscyphus albidus · Ash dieback

\section{Introduction}

Hymenoscyphus fraxineus (formerly known as H. pseudoalbidus) is the causal agent of European ash dieback, a severe disease affecting common ash (Fraxinus excelsior) in Europe (Queloz et al. 2011; Gross et al. 2014a; Baral et al. 2014). Investigations of old herbarium specimens from 1990 and of freshly collected material originating from Japan suggest that the fungus originates in East Asia (Hosoya et al. 1993; Zhao et al. 2012). Indeed, subsequently, the fungus was also reported in East China (Zheng and Zhuang 2013), East Russia (Marčiulynienè et al. 2013) and Korea (Han et al. 2014). A Europe-wide population genetic study showed a severe genetic bottleneck among European $H$. fraxineus populations and supported the alien invasive species hypothesis (Gross et al. 2014b). Until now, a pathogenic behavior of $H$. fraxineus in its native range has not been reported (Zhao et al. 2012; Zheng and Zhuang 2013).

The clade containing $H$. fraxineus currently includes three additionally related species (hereafter referred to as 
the $H$. fraxineus group): Hymenoscyphus albidus, which is native to Europe, is a sister species of $H$. fraxineus (Queloz et al. 2011). It is supposed that H. albidus, which is apparently avirulent on F. excelsior (Husson et al. 2011; Gross et al. 2014a), will be replaced by $H$. fraxineus during the course of the epidemic in Europe (McKinney et al. 2012; see Fig. 23 in Baral and Bemmann 2014). Hymenoscyphus albidoides was derived from rotten leaves of Picrasma quassioides in China (Zheng and Zhuang 2013). Phylogenetic analyses placed this species alongside $H$. albidus and $H$. fraxineus - it is differentiated from the former by croziers at the ascus base and from the latter by the shape of crystals in the stipe-base tissue and the outer covering layer (Zheng and Zhuang 2013). No data on the pathogenicity of $H$. albidoides is available to date. Recently, Hymenoscyphus linearis was derived from petioles of Fraxinus platypoda from Japan (Gross et al. 2014, accepted article). In the phylogeny, $H$. linearis is the most ancestral species in the $H$. fraxineus group but morphological separation proved tedious. In the ascus base showing simple septa, $H$. linearis matches $H$. albidus. The only easily recognizable differentiating characteristic is the distinctive linear shape of the pseudosclerotial plate (sensu Gross and Holdenrieder 2013) that forms on the petioles. Inoculation studies have proven that the species is harmless on F. platypoda and F. excelsior, but there might be a risk of hybridization, especially because $H$. linearis forms a similar anamorph to $H$. fraxineus (Gross et al. 2014, accepted article).
Knowledge of the native distribution range of introduced pathogenic species is crucial for accurately locating their origin (Dlugosch and Parker 2008). Knowing the host range is also important since different hosts could serve as reservoirs for the pathogen and this might be a consideration for quarantine measures (Stergiopoulos and Gordon 2014). Finally, knowledge of closely related and/or cryptic species is of great importance for avoiding pathogen virulence evolution through hybridization (Olson and Stenlid 2002).

In the present study, we re-examined putative $H$. fraxineus specimens of Han et al. (2014) and five additional collections from Korean F. mandshurica and Fraxinus chinensis subsp. rhynchophylla (synonym $=$ Fraxinus rhynchophylla $)$. Our aims were to investigate the host and distribution range of H. fraxineus and to detect any possible cryptic species.

\section{Methods}

Fungal material and DNA extraction

A total of nine Korean collections of putative $H$. fraxineus on pseudosclerotial leaf rachises and petioles (collectively referred to as petioles hereafter) containing numerous ascomata were studied (Table 1, Fig. 1). Depending on the quantity of the collection, one to three single apothecia of each collection were randomly sampled and DNA was extracted as described by Grünig et al. (2003). In addition, collection KUS-F52847

Table 1 Putative Korean H. fraxineus collections analyzed in this study

\begin{tabular}{|c|c|c|c|c|c|c|c|}
\hline $\mathrm{Nr}$. & Specimen name & Collection date & Location & Latitude $\left({ }^{\circ} \mathrm{N}\right)$ & Longitude $\left({ }^{\circ} \mathrm{E}\right)$ & $\begin{array}{l}\text { Altitude } \\
\text { (m.a.s.1.) }\end{array}$ & Substrate \\
\hline 1 & KUS-F52255 ${ }^{\mathrm{a}}$ & 14.08 .2008 & Mt. Taegi, Pyeongchang & 37.590278 & 128.279722 & 1,037 & $\begin{array}{l}\text { petioles of } F \text {. chinensis } \\
\text { subsp. rhynchophylla }\end{array}$ \\
\hline 2 & KUS-F52355 ${ }^{\mathrm{a}}$ & 23.09.2008 & Supchaewon, Hoengseong & 37.390000 & 128.294167 & 1,097 & rotten leaves of $F$ mandshurica \\
\hline 3 & KUS-F52542 & 15.07.2009 & $\begin{array}{r}\text { Seolmaejae recreation } \\
\text { forest, Yangpyeong }\end{array}$ & 37.553889 & 127.503333 & 305 & $\begin{array}{l}\text { petioles of } F \text {. chinensis subsp. } \\
\text { rhynchophylla }\end{array}$ \\
\hline 4 & KUS-F52552 & 15.07.2009 & $\begin{array}{l}\text { Sanuem recreation forest, } \\
\text { Yangpyeong }\end{array}$ & 37.602500 & 127.598333 & 207 & $\begin{array}{l}\text { petioles of } F \text {. chinensis subsp. } \\
\text { rhynchophylla }\end{array}$ \\
\hline 5 & KUS-F52610 a & 19.08.2009 & $\begin{array}{l}\text { Cheongtaesan recreation } \\
\text { forest, Hoengseong }\end{array}$ & 37.525556 & 128.292778 & 850 & $\begin{array}{l}\text { petioles of } F \text {. chinensis subsp. } \\
\quad \text { rhynchophylla }\end{array}$ \\
\hline 6 & KUS-F52613 ${ }^{\mathrm{a}}$ & 20.08 .2009 & Supchaewon, Hoengseong & 37.390278 & 128.292778 & 1,040 & $\begin{array}{l}\text { petioles of } F \text {. chinensis subsp. } \\
\text { rhynchophylla }\end{array}$ \\
\hline 7 & KUS-F52742 & 12.07.2010 & $\begin{array}{l}\text { Darian valley in Mt. } \\
\text { Sobeak National Park, } \\
\text { Danyang }\end{array}$ & 36.962778 & 128.423333 & 385 & $\begin{array}{l}\text { petioles of } F \text {. chinensis subsp. } \\
\text { rhynchophylla }\end{array}$ \\
\hline 8 & KUS-F52784 & 27.08 .2010 & $\begin{array}{l}\text { Woljeongsa Temple in } \\
\text { Mt. Odae National } \\
\text { Park, Pyeongchang }\end{array}$ & 37.731667 & 128.594444 & 662 & $\begin{array}{l}\text { petioles of } F \text {. chinensis subsp. } \\
\text { rhynchophylla }\end{array}$ \\
\hline 9 & KUS-F52847 & 04.08 .2012 & $\begin{array}{l}\text { Deokduwon-ri, } \\
\text { Seo-myeon, Chuncheon }\end{array}$ & 37.864167 & 127.658056 & 120 & $\begin{array}{l}\text { petioles of } F \text {. chinensis subsp. } \\
\text { rhynchophylla }\end{array}$ \\
\hline
\end{tabular}

All collected by Jae-Gu Han. Species identification of the collections is given in Table 2

${ }^{\text {a }}$ Collections were already analyzed by Han et al. (2014) and identified as $H$. fraxineus 
Fig. 1 Map of South Korea showing the position of all collections treated in this study. Species identification based on our analysis is given for each collection

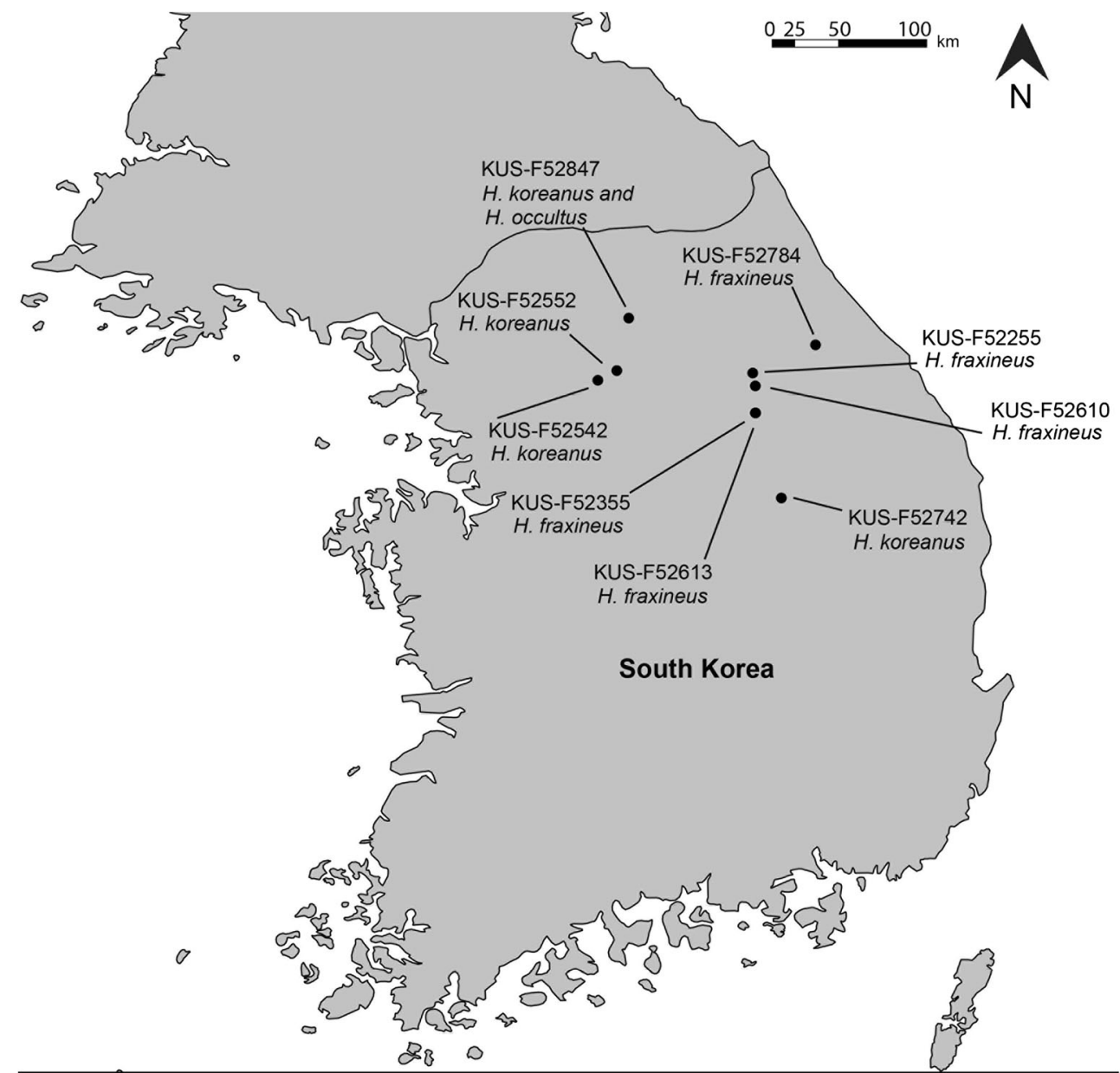

was still alive and 25 isolations from petioles were carried out as described in Gross and Holdenrieder (2013). From the successful fungal isolations (Table 2), mycelium was lyophilized and DNA was extracted with the NucleoSpin 96 plant II kit (Macherey-Nagel, Düren, Germany) using the hexadecyltrimethylammonium bromide (CTAB)-based lysis buffer PL1 according to the protocol of the manufacturer. All collections were deposited in the Korea University Herbarium (KUS), Seoul, South Korea.

Initial ITS screening for species identification

Of all Korean samples, the internal transcribed spacers (ITS) 1 and 2 of the rDNA (primers ITS1 and ITS4 of White et al. 1990) were amplified by a standard polymerase chain reaction (PCR) protocol, followed by sequencing with the ITS4 primer as described in Gross et al. (2014b). The sequences were manually edited in Geneious v.6.1.3 and a multiple alignment (using the ClustalW tool of Geneious set to default parameters) was carried out with (i) all new sequences (ii) a reference strain of $H$. fructigenus and (iii) several known species of the H. fraxineus group available in GenBank; i.e., H. albidus,
$H$. fraxineus, $H$. albidoides and $H$. linearis. Three distinct ITS groups were identified in Korean samples (Table 2).

Phylogenetic analysis

Based on the ITS screening, 14 Korean samples were chosen for a more detailed phylogenetic analysis using four different sequence markers (Table 2). For all of these samples, the ITS, as well as a partial fragment of the actin gene, the calmodulin gene and the EF1- $\alpha$ gene, were amplified and sequenced bidirectionally in accordance with the procedure described in Gross et al. (2014b). The primers and primer sources used to amplify Korean samples were as follows: ITS, primers ITS1 and ITS4 (White et al. 1990); actin, Act-512F (Carbone and Kohn 1999) and Hp_actin_r(Gross et al. 2014b); calmodulin, Cal-228F and Cal-737R (Carbone and Kohn 1999) for ITS group I and II, Cal-228F and Hym Cal R (Gross et al. 2014, accepted article) for ITS group III; EF1- $\alpha$, EF1-728F (Carbone and Kohn 1999) and EF1- $\alpha$ R2 (Gross et al. 2014, accepted article) for ITS group I, EF1-728F and EF1- $\alpha$ R (Gross et al. 2014, accepted article) for ITS group II and III. Sequences were edited in Geneious v6.1.3, primer sequences were removed, and pairwise alignments of the 
Table 2 List of samples for initial ITS screening (ITS-S) and subsequent phylogenetic analysis (Phyl-A)

\begin{tabular}{|c|c|c|c|c|c|c|c|c|c|}
\hline Sample name & Origin $^{a}$ & Type PS & Study & ITS group & Species & ITS & Calmodulin & $E F 1-\alpha$ & Actin \\
\hline KUS-F52255_1 & 1 & & ITS-S/Phyl-A & I & H. fraxineus & KP068053 & KP068025 & KP068039 & KP068011 \\
\hline KUS-F52255_2 & 1 & & ITS-S & I & H. fraxineus & KP068080 & NA & NA & NA \\
\hline KUS-F52355_1 & 1 & & ITS-S/Phyl-A & I & H. fraxineus & KP068051 & KP068023 & KP068037 & KP068009 \\
\hline KUS-F52610_1 & 1 & & ITS-S & I & H. fraxineus & KP068076 & NA & NA & NA \\
\hline KUS-F52610_2 & 1 & & ITS-S & I & H. fraxineus & KP068075 & NA & NA & NA \\
\hline KUS-F52613_1 & 1 & & ITS-S/Phyl-A & I & H. fraxineus & KP068055 & KP068027 & KP068041 & KP068013 \\
\hline KUS-F52784_1 & 1 & & ITS-S/Phyl-A & I & H. fraxineus & KP068056 & KP068028 & KP068042 & KP068014 \\
\hline KUS-F52784_2 & 1 & & ITS-S & I & H. fraxineus & KP068073 & NA & NA & NA \\
\hline KUS-F52542_1 & 1 & & ITS-S & II & H. koreanus & KP068079 & NA & NA & NA \\
\hline KUS-F52542_2 & 1 & & ITS-S & II & H. koreanus & KP068078 & NA & NA & NA \\
\hline KUS-F52552_1 & 1 & & ITS-S/Phyl-A & II & H. koreanus & KP068054 & KP068026 & KP068040 & KP068012 \\
\hline KUS-F52552_2 & 1 & & ITS-S & II & H. koreanus & KP068077 & NA & NA & NA \\
\hline KUS-F52742_1 & 1 & & ITS-S/Phyl-A & II & H. koreanus & KP068052 & KP068024 & KP068038 & KP068010 \\
\hline KUS-F52742_2 & 1 & & ITS-S & II & H. koreanus & KP068074 & NA & NA & NA \\
\hline KUS-F52847_Fk1 & 1 & & ITS-S & II & H. koreanus & KP068067 & NA & NA & NA \\
\hline KUS-F52847_Fk2 & 1 & & ITS-S & II & H. koreanus & KP068066 & NA & NA & NA \\
\hline KUS-F52847_Fk3 & 1 & & ITS-S & III & H. occultus & KP068065 & NA & NA & NA \\
\hline KUS-F52847_01 & 2 & A & ITS-S/Phyl-A & II & H. koreanus & KP068057 & KP068029 & KP068043 & KP068015 \\
\hline KUS-F52847_04 & 2 & A & ITS-S/Phyl-A & II & H. koreanus & KP068059 & KP068031 & KP068045 & KP068017 \\
\hline KUS-F52847_05 & 2 & $\mathrm{~A}$ & ITS-S/Phyl-A & II & H. koreanus & KP068060 & KP068032 & KP068046 & KP068018 \\
\hline KUS-F52847_06 & 2 & $\mathrm{~A}$ & ITS-S/Phyl-A & II & H. koreanus & KP068061 & KP068033 & KP068047 & KP068019 \\
\hline KUS-F52847_08 & 2 & A & ITS-S & II & H. koreanus & KP068072 & NA & NA & NA \\
\hline KUS-F52847_12 & 2 & A & ITS-S & II & H. koreanus & KP068071 & NA & NA & NA \\
\hline KUS-F52847_15 & 2 & $\mathrm{~A}$ & ITS-S & II & H. koreanus & KP068070 & $\mathrm{NA}$ & NA & NA \\
\hline KUS-F52847_18 & 2 & A & ITS-S & II & H. koreanus & KP068069 & NA & NA & NA \\
\hline KUS-F52847_19 & 2 & A & ITS-S & II & H. koreanus & KP068068 & NA & NA & NA \\
\hline KUS-F52847_03 & 2 & $\mathrm{~B}$ & ITS-S/Phyl-A & III & H. occultus & KP068058 & KP068030 & KP068044 & KP068016 \\
\hline KUS-F52847_22 & 2 & $\mathrm{~B}$ & ITS-S/Phyl-A & III & H. occultus & KP068062 & KP068034 & KP068048 & KP068020 \\
\hline KUS-F52847_24 & 2 & $\mathrm{~B}$ & ITS-S/Phyl-A & III & H. occultus & KP068063 & KP068035 & KP068049 & KP068021 \\
\hline KUS-F52847_25 & 2 & $\mathrm{~B}$ & ITS-S/Phyl-A & III & H. occultus & KP068064 & KР068036 & KP068050 & KP068022 \\
\hline HMAS 264140 & 3 & & Phyl-A & & H. albidoides & KF188722 & KF188732 & NA & NA \\
\hline Cas_01 & 3 & & Phyl-A & & H. albidus & GU586882 & GU586937 & GU586956 & KJ511015 \\
\hline Lav_01 & 3 & & Phyl-A & & H. albidus & GU586884 & GU586938 & GU586957 & KJ511047 \\
\hline Qui_01 & 3 & & Phyl-A & & H. albidus & GU586887 & GU586939 & GU586958 & KJ511048 \\
\hline Aubo_03 & 3 & & Phyl-A & & H. fraxineus (Europe) & KJ511173 & KJ511069 & KJ511121 & KJ511012 \\
\hline Kore_06 & 3 & & Phyl-A & & H. fraxineus (Europe) & KJ511201 & KJ511097 & KJ511149 & KJ511043 \\
\hline CBS_122504 & 3 & & Phyl-A & & H. fraxineus (Europe) & FJ597975 & GU586951 & GU586970 & KJ511016 \\
\hline Suga_27 & 3 & & Phyl-A & & H. fraxineus (Japan) & KJ511208 & KJ511104 & KJ511156 & KJ511052 \\
\hline Suga_28 & 3 & & Phyl-A & & H. fraxineus (Japan) & KJ511209 & KJ511105 & KJ511157 & KJ511053 \\
\hline Suga_44 & 3 & & Phyl-A & & H. fraxineus (Japan) & KJ511215 & KJ511111 & KJ511163 & KJ511059 \\
\hline Hokk_24 & 3 & & Phyl-A & & H. fraxineus (Japan) & KJ511194 & KJ511090 & KJ511142 & KJ511036 \\
\hline Hokk_27 & 3 & & Phyl-A & & H. fraxineus (Japan) & KJ511195 & KJ511091 & KJ511143 & KJ511037 \\
\hline Hokk_74 & 3 & & Phyl-A & & H. fraxineus (Japan) & KJ511197 & KJ511093 & KJ511145 & KJ511039 \\
\hline CBS_650.92 & 3 & & Phyl-A & & H. fructigenus & GU586933 & GU586952 & GU586971 & KJ511017 \\
\hline Chic_01 & 3 & & Phyl-A & & H. linearis & KM114521 & KM114471 & KM114496 & KM114446 \\
\hline Chic_03 & 3 & & Phyl-A & & H. linearis & KM114523 & KM114473 & KM114498 & KM114448 \\
\hline
\end{tabular}

The origin of the samples and the type of pseudosclerotium (PS) of the isolations from KUS-F52847 are given. In addition, the inferred phylogenetic group and GenBank accession numbers for each sample and genetic marker are given. Accession numbers of sequences generated in this study are in bold. NA indicates non-available data

${ }^{a}$ 1, DNA isolation from single, dried apothecium; 2, DNA isolation from culture; 3, DNA sequences extracted from GenBank 
corresponding forward and reverse sequences of each sample were carried out. The dataset for phylogenetic analysis was supplemented with published sequences (available in GenBank) of all members of the $H$. fraxineus group and H. fructigenus was used as the outgroup (Table 2). A multiple-sequence alignment was performed for each marker using the ClustalW tool implemented in Geneious, set to the default parameters. Newly-generated sequences were deposited in GenBank and accession numbers are listed in Table 2.

Prior to phylogenetic analysis, duplicate sequences were removed from the alignments and ambiguously aligned sites were removed in a standardized way using Gblocks web v0.91b (http://molevol.cmima.csic.es/castresana/Gblocks server.html). Less stringent options were applied, allowing for smaller final blocks, gaps in the final alignment and less strict flanking positions. Each single locus alignment was subject to Bayesian inference (BI) phylogenetic analysis in MrBayes v 3.2.2 (Ronquist et al. 2012). The general time reversible (GTR) nucleotide substitution model was applied with gamma-distributed rate variation across sites and a proportion of invariable sites. The two parallel Markov chain Monte Carlo (MCMC) analysis runs were performed with four chains for 2,000,000-3,000,000 generations while trees were sampled every 500 generations and $25 \%$ of the total generations were discarded (burn-in). Convergence of the two parallel runs was verified by ensuring that the standard deviation of the split frequency fell below 0.01 and by verifying that the posterior probabilities over the number of generations reached a stable phase through use of the software tracer (Drummond and Rambaut 2008). Trees were visualized using FigTree v 1.4 (http://tree.bio.ed.ac.uk/software/figtree/).

PartitionFinder v 1.1.0 (Lanfear et al. 2012) software was used to find the optimal nucleotide substitution model for each marker in the combined alignment. Consequently, we partitioned the combined alignment according to the alignment lengths of each single marker. The program was run on the 'greedy' search algorithm, with 'linked' branch-lengths, and the best model (restricted to available models in MrBayes or RAxML, respectively) was selected based on the Bayesian information criterion (BIC). Subsequently, the combined dataset was subject to BI analysis as described before, but with the $\mathrm{K} 80+\mathrm{I}$ (proportion of invariable sites) substitution model for the ITS partition and the $\mathrm{K} 80+\mathrm{G}$ (gamma-distributed rate variation across sites) substitution model for the actin, calmodulin and EF1- $\alpha$ partitions. Maximum likelihood (ML) analysis of the combined alignment was performed with the software RAxML v 7.7.7 (Stamatakis 2006). One thousand initial independent $\mathrm{ML}$ inferences were performed on the partitioned alignment file using a GTR $+\mathrm{G}$ model (for all markers), while support values based on 1,000 bootstrap replicates were drawn on the tree with the best
ML score. All trees and alignments were deposited in Treebase (TreeBase ID: 16600).

Investigating the ability to produce ascocarps in culture

Spontaneous ascocarp development in culture has already been observed in H. linearis (Gross et al. 2014, accepted article). A subsequent experiment was set up to test whether other species of the $H$. fraxineus group are also able to self-fertilize and produce the teleomorph in culture. Three different isolates of ITS group II, ITS group III and H. albidus, two single spore isolates of $H$. linearis (positive control) and two isolates with alternating mating types of $H$. fraxineus were selected for the experiment (Table 4). The two chosen $H$. fraxineus strains (Abts_22 and Abts_33) mated successfully in a previous crossing experiment on petioles (see Gross et al. 2012). All strains were freshly cultivated on ash leaf malt-extract agar (AMA) (Gross et al. 2014b) in polystyrene petri dishes (Greiner Bio-One, Frickenhausen, Germany) and incubated at $20{ }^{\circ} \mathrm{C}$ in an incubator in the dark. The two $H$. fraxineus strains were inoculated approximately $4 \mathrm{~cm}$ apart from each other on a single agar plate. After 23 days, cultures were removed and transferred to an incubator producing near-UV light (Leach 1971) for $12 \mathrm{~h} \mathrm{day}^{-1}$ in the light at $19.5^{\circ} \mathrm{C}$, followed by $12 \mathrm{~h} \mathrm{day}^{-1}$ in the dark at $16.5^{\circ} \mathrm{C}$. The cultures were checked regularly for the appearance of ascocarps and, when they emerged, germination of spores was verified on AMA plates.

\section{Microscopic examinations}

Studies were carried out on fresh ascocarps of the strains KUS-F52847_01 (ITS group II) and KUS-F52847_03 (ITS group III), both produced on AMA plates. A Zeiss Axiophot microscope was used and pictures were taken with the integrated camera AxioCam MRc5. Observations were made in lactic acid (LA), $\mathrm{H}_{2} \mathrm{O}$ and Lugol's reagent. All measurements were taken from material mounted in LA, with an enlargement factor of $1,000 \times$, by using differential interference contrast. The mean and standard errors were calculated whenever 10 20 measurements were obtained. The morphology of herbarium collections was not studied because of the possibility of multiple species appearing in single collections (see below).

\section{Results}

Fungal isolations and ITS screening

Thirteen of the isolations from different petioles of the vital collection KUS-F52847 proved successful (Table 2). Two types of pseudosclerotia were observed on the petioles during the isolation process: Type A was indistinguishable from 
$H$. albidus or $H$. fraxineus; i.e., the black pseudosclerotial plate surrounded and enclosed large parts of the petiole. Type B was distinctly thinner and only superficial in crosssection view, however, it was irregularly-shaped and only enclosed a small fraction of the petiole in surface-view (making it tedious to isolate the fungus from inside the pseudosclerotium).

Regarding all collections from Korea (Tables 1 and 2), three different ITS groups were identified. Group I was highly similar or identical to $H$. fraxineus from Japan, group II (described here as $H$. koreanus) was similar to $H$. albidus from Europe (99.6 \% identical, 2-bp difference) and group III (described here as $H$. occultus) was distinct from all reference Hymenoscyphus species, showing the highest affinity to group II with a sequence identity of $95.8 \%$ (Table 3 ). Altogether, five collections belonged to group I, three collections to group II, and the fresh collection KUS-F52847 was composed of group II and III genotypes, whereas the two groups could be detected in isolates and genotyped ascocarps of that collection (Table 2). The isolates of ITS group II, from KUS-F52847, consistently originated from type A poseudosclerotia, while those belonging to group III were consistently derived from type B pseudosclerotia.

Phylogenetic analysis using four sequence markers

Fourteen samples originating from Korean collections and 16 reference samples of $H$. albidus, $H$. fraxineus (from Europe and Japan), H. linearis, $H$. albidoides, and $H$. fructigenus (outgroup) were selected for a more detailed phylogenetic analysis using four different sequence markers. For H. albidoides, only the sequence of the ITS and calmodulin could be used as it was not possible to obtain the herbarium specimen of this species. In addition, for samples KUSF52784 1 and KUS-F52255 1, the actin sequence was truncated at the $3^{\prime}$ and $5^{\prime}$ end because a poly-C stretch in the intron hampered the sequencing. The alignment lengths of single and combined markers were as follows (in [bp], lengths after the removal of ambiguously aligned sites in brackets): ITS $=485$
(481); actin $=257$ (241); EF1- $\alpha=555$ (549); calmodulin $=390$ (375); and combined $=1,687(1,646)$. Phylogenetic analysis using the single sequence markers revealed concordant groupings, except for isolate KUS-F52613_1; whereas this isolate normally grouped with $H$. fraxineus, it formed a separate branch basal to $H$. albidus in the EF1- $\alpha$ phylogeny. Since only minor incongruences were found between single tree topologies, the sequences were combined. The combined phylogenetic analysis was congruent with the single gene trees. Samples of ITS group I fell in the clade containing $H$. fraxineus strains, samples of ITS group II formed a separate branch next to $H$. albidus, with maximal statistical support, and samples of ITS group III also formed a separate branch with maximal support basal to $H$. albidus, $H$. albidoides and $H$. fraxineus. Based on the phylogenetic analysis, ITS group II and III represent novel species within the genus Hymenoscyphus, whereas group I represents $H$. fraxineus.

Ability of $H$. fraxineus group species to produce ascocarps in culture

The ability to produce ascocarps in culture was confirmed for all species except for $H$. fraxineus (Table 4). However, only one of three isolates of $H$. albidus produced the teleomorph and only one ascocarp was produced by this culture. Ascoma initials (short, erect stipes without a discus) were observed 12 days after incubation at near-UV light on one culture belonging to ITS group II and two cultures belonging to ITS group III isolates. The maturity of the ascocarps, defined as the ability to produce viable ascospores, was reached between 41 and 71 days after incubation at near-UV light. Sporulation and germination of ascospores were verified on AMA plates and could be confirmed for all ascocarp-forming species. However, the ascocarps of culture KUS-F52847 08 remained small and did not eject any ascospores, and the ascospores of culture KUS-F52847 04 did not germinate. Remarkably, the first ascospore germination test failed in all but isolate KUSF52847 06, whereas only the second or third germination test was fully successful (data not shown). The ascocarps of ITS

Table 3 Distance matrix (\% identical positions in the ITS alignment) of several closely related Hymenoscyphus members and H. fructigenus based on one representative strain (given in parentheses)

H.fructigenus H.fraxineus H.albidus H.albidoides H.linearis H.fraxineus H.koreanus H.occultus

H. fructigenus (CBS_650.92)

H. fraxineus (CBS_122504)

H. albidus (Cas_01)

H. albidoides (HMAS_264140)

H. linearis (Chic_01)

H. fraxineus (KUS-F52255_1)

H. koreanus (KUS-F52847_01)

H. occultus (KUS-F52847_03)
91.5

91.7

90.6

88.2

91.3

91.7

91.5

\section{7}

$96.2 \quad 96.4$

$92.6 \quad 93.2$

$99.2 \quad 97.7$

$97.9 \quad 99.6$

$94.8 \quad 95.4$
91.5

$96.2 \quad 92.4$

$\begin{array}{lll}96.8 & 93.2 & 97.9\end{array}$

$\begin{array}{llll}94.5 & 93.4 & 94.6 & 95.8\end{array}$ 
Table 4 The ability of spontanous ascocarp-production on agar medium of all members of the $H$. fraxineus group (except $H$. albidoides)

\begin{tabular}{llll}
\hline Strains & Species & Apothecia production & Sporulation/germination \\
\hline KUS-F52847_04 & H. koreanus & yellow apothecia & sporulated well but no germination observed \\
KUS-F52847_06 & H. koreanus & yellow apothecia & $100 \%$ germination \\
KUS-F52847_08 & H. koreanus & few small yellow apothecia on agar inoculum & no sporulation observed \\
KUS-F52847_03 & H. occultus & white apothecia & $100 \%$ germination \\
KUS-F52847_24 & H. occultus & white apothecia & $100 \%$ germination \\
KUS-F52847_25 & H. occultus & white apothecia & $100 \%$ germination \\
090802.2 & H. albidus & no apothecia formed & \\
090723.1 & H. albidus & no apothecia formed & $100 \%$ germination \\
Mugg_12 & H. albidus & one cream-white apothecium & \\
Abts_22 \& Abts_33 & H. fraxineus & no apothecia formed & $100 \%$ germination \\
CBS_138754 & H. linearis & yellow apothecia & \\
Chic_fk1_ss2 & H. linearis & no apothecia formed, contaminated & \\
\hline
\end{tabular}

${ }^{\mathrm{a}}$ Isolate from specimen 090802.2, see Queloz et al. 2011

${ }^{\mathrm{b}}$ Isolated by O. Holdenrieder from petioles of $F$. excelsior (23.07.2009) from a sample collected in Ennenda, Switzerland, N 47.043056 E 9.079667

${ }^{\mathrm{c}}$ Isolated by A. Gross from petioles of F. excelsior (2.5.2012) from a sample collected near Muggio, Switzerland, N 45.899583 E 9.046583

${ }^{\mathrm{d}}$ From the same single spore isolation series as CBS_138754, see Gross et al. 2014

group II isolates and $H$. linearis were bright yellow in culture, whereas that of ITS group III were pale white. The single ascocarp of $H$. albidus was cream-white to yellow in color.

ITS group II: Hymenoscyphus koreanus A. Gross \& J.

\section{G. Han, sp. nov.}

\section{MycoBank: MB 810719}

Etymology: Referring to the country of origin, where it was repeatedly detected.

Holotype: A dried culture containing ascomata of isolate KUS-F52847_01, which was isolated from collection KUSF52847, Korea, Deokduwon-ri, Seo-myeon, Chuncheon, 120 m, petioles of Fraxinus chinensis subsp. rhynchophylla, 04.08.2012, collected by J.G. Han. The holotype comprises a dried culture containing many ascomata (same as shown in a living state in Fig. 3b). It is deposited in the Korea University Herbarium (KUS), Seoul, South Korea, KUS-ID: KUSF52851. The same fungal strain was selected as a type strain and a living duplicate was deposited in the CBS culture collection in Utrecht, Netherlands (CBS-ID: CBS 139466)

\section{Teleomorph:}

Apothecia: nail-shaped with a $0.65-1 \mathrm{~mm}$ long and $0.3-$ $0.4 \mathrm{~mm}$ wide stipe and a disc diameter of (0.8-) 1.6-2.8 mm; arising from superficial pseudosclerotial plates on AMA; disc concave to convex, depending on the age, and cream-white to light yellow in color (Fig. 3b); surfaces of the hymenium, stipe and receptacle are smooth, the stipe has a brown to black basal collar, containing irregularly-shaped single crystals in crosssection (Fig. 3f). Asci clavate, short stipitate (Fig. 3i), 82$105 \mu \mathrm{m}(\varnothing 93.65, \pm 2.29)$ long, 7.5-9.5 $\mu \mathrm{m}(\varnothing 8.65, \pm 0.24)$ wide at the widest point, eight-spored; apical ring blue in Lugol's reagent, Hymenoscyphus-type (Fig. 3d) (see Baral 1987), without croziers (Fig. 3e). Ascospores biseriate even in dead state (Fig. 3c), slightly "scutuloid" (like spores of H. scutula), broadly rounded (profile view) and more or less beaked (side view) at one end, more or less narrowed and almost pointed at the other end, straight in profile view, slightly arcuate in side view, slightly waisted in the middle, hyaline, non-septate, with two or more large and some smaller, globose oil drops (living state in $\mathrm{H}_{2} \mathrm{O}$, Fig. $3 \mathrm{~g}$ ), or 1-2 confluent, partly elongated oil droplets in lactic acid (Fig. 3c), without setulae, 13.5-20 $\mu \mathrm{m}(\varnothing 16.53, \pm 0.79) \times 3.5-4.5 \mu \mathrm{m}(\varnothing 3.9, \pm 0.11)$ in LA, becoming slightly brown and often one-septate upon germination on AMA, forming 1-2 germ tubes (Fig. 31). Paraphyses cylindrical, hyaline, 2-3 $\mu \mathrm{m}$ thick, upper septum 37-64 $\mu \mathrm{m}$ below apex, strongly refractive vacuolar bodies observed when mounted in $\mathrm{H}_{2} \mathrm{O}$ (Fig. 3h). Ectal excipulum of textura prismatica with more or less rectangular cells, $10-30 \times 3-6 \mu \mathrm{m}$, cortical cells hyaline or slightly yellow (Fig. 2j). Medullary excipulum hyaline, of textura intricata, outer part of textura porrecta, cells 27-37×2.5-3.5 $\mu \mathrm{m}$ (Fig. 3k).

Anamorph (KUS-F52847_08, Fig. 3m-p): Only nonsporulating conidiogenous structures were observed. The phialids occurred singly or were arranged in loose aggregations, 1-4-septate at the base, 20.75-29.5 $\mu \mathrm{m}$ long, light brown but gradually turning hyaline towards the tip; venter

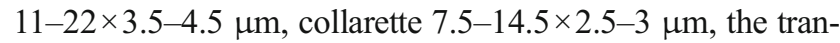
sition between the collarette and venter was often gradual, sometimes abrupt, often with a conspicuous septum inbetween (Fig. 3o). Mostly two, sometimes one, small oil drops were present in the apical part.

Remarks:

The teleomorph of $H$. koreanus revealed the same gross morphology compared to the other species within the 


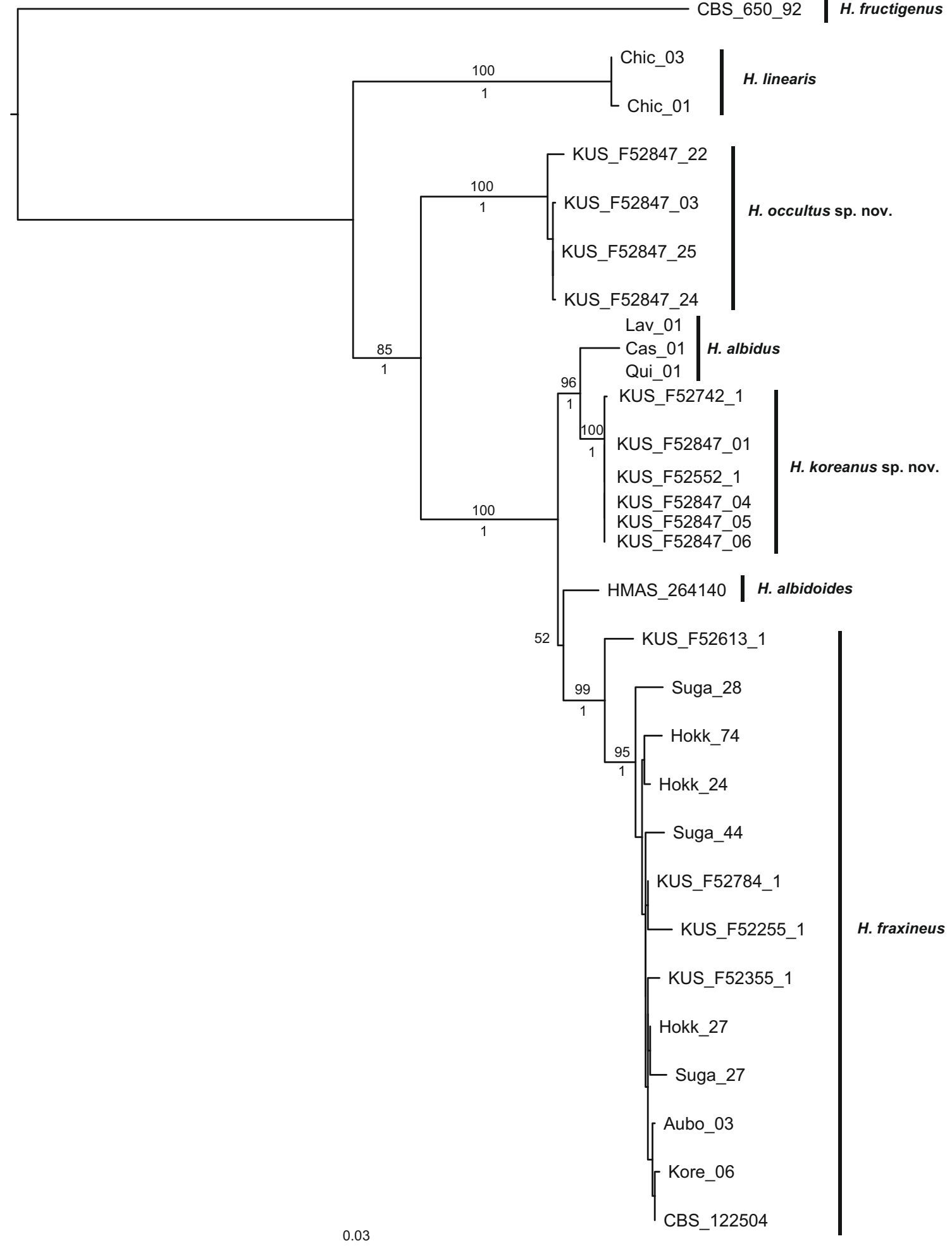

Fig. 2 Phylogenetic analysis of the concatenated multiple sequence alignment of markers ITS, EF1- $\alpha$, calmodulin and actin. Bootstrap percentages of ML phylogenetic analysis are given above the branches, while posterior probabilities of Bayesian phylogenetic analysis are given below the branches. The scale bar represents the number of substitutions

$H$. fraxineus group. It differs from $H$. occultus by having smaller asci, from $H$. fraxineus and $H$. albidoides by the per site. The clades of the two new species, H. occultus and H. koreanus, are supported by maximal statistical values. Strains with identical sequences were omitted from the alignment and manually added to the tree post analysis

absence of croziers at the ascus base, and from $H$. albidus by having single crystals in the apothecial stipe and the 

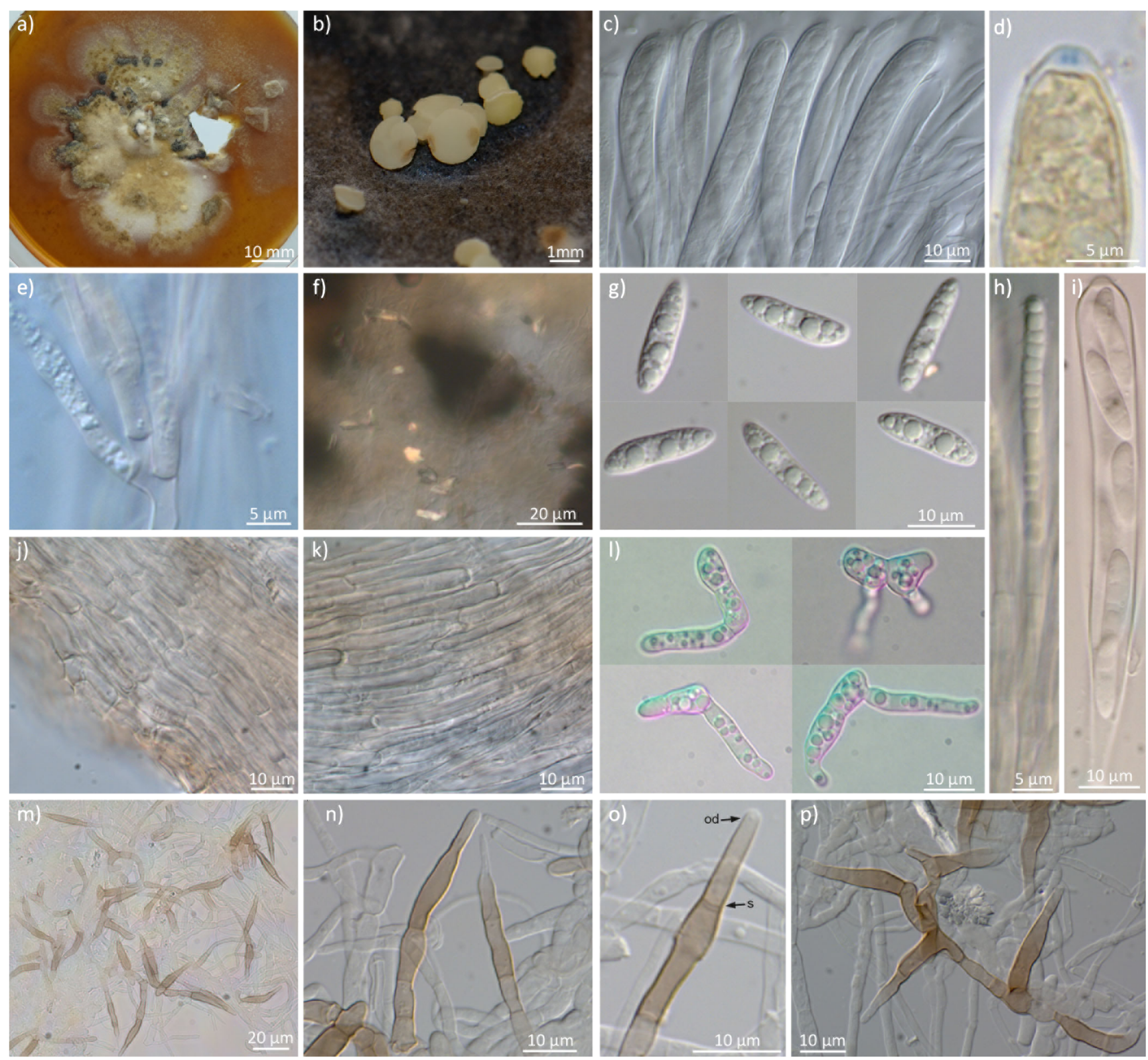

Fig. 3 Morphology of H. koreanus sp. nov., teleomorph (KUS-F52847 01 ) and anamorphic structures (KUS-F52847_08) produced in culture. Microscopic slides were prepared in LA if not specified otherwise. a 41day-old culture on AMA showing irregular growth at the margin and zones of different colors, while black areas represent pseudosclerotial structures. b Apothecia emerging from the pseudosclerotial plate (PSP) on AMA. c Hymenium. d Ascus tip showing the amyloid ring in Lugol's reagent. e Ascus base showing the absence of croziers. f Irregularly shaped crystals in the stipe base. g Ascospores mounted in $\mathrm{H}_{2} \mathrm{O}$ (living state). h Paraphysis in $\mathrm{H}_{2} \mathrm{O}$ (living state) showing strongly refractive

vacuolar bodies (not visible in LA). i Entire ascus. $\mathbf{j}$ Ectal excipulum. $\mathbf{k}$ Medullary excipulum (outer part). I Germinating ascospores showing septation and one to two germ tubes mounted in $\mathrm{H}_{2} \mathrm{O}$ (living state). $\mathbf{m}$ Anamorphic structures: Brown phialides formed in the aerial mycelium on an AMA culture. $\mathbf{n}$. Phialophore bearing a single phialide with stunted development. o Phialide showing the frequently observed septum (s, maybe due to the de-bonding of the first forming phialoconidium) between the venture and collarette and a small oil drop (od) in the apical part. p Phialophore showing five phialides. Phialoconidia were not observed

formation of an anamorph-like structure in culture. No unique nucleotide positions were found in the ITS sequence of $H$. koreanus compared to the other species of the $H$. fraxineus group (Online Resource 1). However, it differs from $H$. albidus in positions $80(\mathrm{~T})$ and $358(\mathrm{~T})$ and from $H$. albidoides in positions 74 (T), 120(T),
364(C), 439(A) and 433(A). In the calmodulin sequence alignment (Online Resource 2), it revealed the unique nucleotide positions 59(A), 152 (A) and a characteristic deletion of positions 33-41.

ITS group III: Hymenoscyphus occultus A. Gross \& J. G. Han, sp. nov. 


\section{MycoBank: MB 810718}

Etymology: Referring to the rarity of the species in Korea and the high chance that the species is overlooked.

Holotype: A dried culture containing apothecia of isolate KUS-F52847 03 isolated from collection KUS-F52847, Korea, Deokduwon-ri, Seo-myeon, Chuncheon, 120 m, petioles of Fraxinus chinensis subsp. rhynchophylla, 04.08.2012, collected by J.G. Han. The holotype comprises a dried culture containing many apothecia (same as that shown in a living state in Fig. 4b). It is deposited in the Korea University Herbarium (KUS), Seoul, South Korea, under the KUS-ID: KUS-F52850. The same strain was selected as the type strain and a living duplicate was deposited in the CBS culture collection in Utrecht, Netherlands (CBS-ID: CBS 139469)
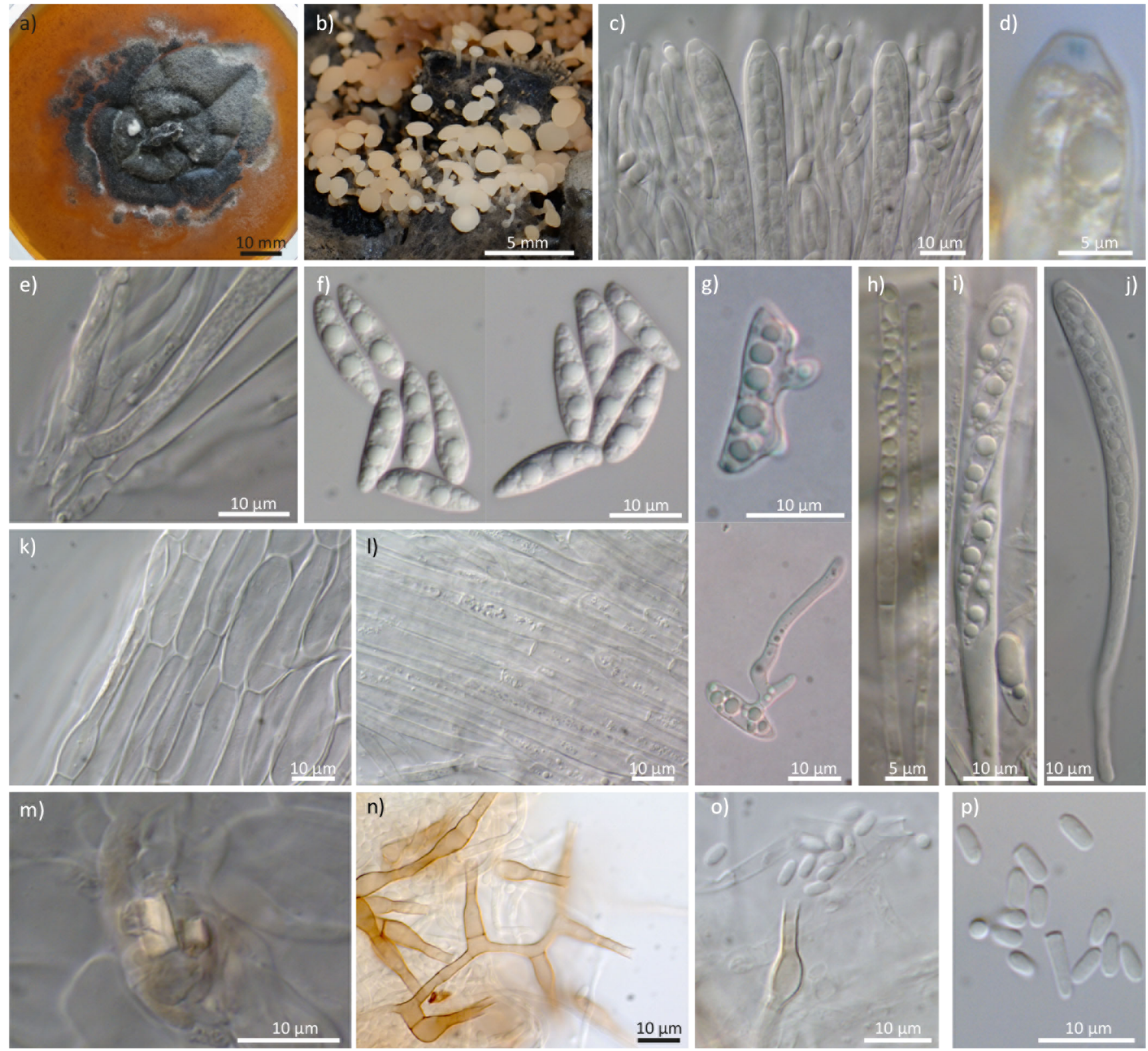

Fig. 4 Morphology of H. occultus sp. nov., teleomorph and anamorph produced in culture of KUS-F52847_03. Microscopic slides were prepared in LA if not specified otherwise. a 43-day- old culture that formed a laminary, superficial pseudosclerotial plate (PSP) on AMA. b Apothecia emerging from PSP formed on an AMA culture after incubation at near-UV light. $\mathbf{c}$ Vertical section through the hymenium showing asci and paraphyses. $\mathbf{d}$ Ascus tip in Melzer's reagent showing a slight bluing of the pore. e Ascal bases without croziers. f Living ascospores in $\mathrm{H}_{2} \mathrm{O}$ showing the 'scutuloid' shape. $\mathrm{g}$ Germinating ascospores on AMA (living state in $\mathrm{H}_{2} \mathrm{O}$ ), forming one or two germ tubes, with or without a septum in the middle. h Paraphyses in $\mathrm{H}_{2} \mathrm{O}$ (living state) showing strongly refractive vacuolar bodies (absent in LA). i Dead ascus in $\mathrm{H}_{2} \mathrm{O}$ containing living spores. j Entire distinctly long stipitate ascus. $\mathbf{k}$ Ectal excipulum. I Medullary excipulum (outer part). $\mathbf{m}$ Crystals found in the stipe base. n Anamorph: Phialophore bearing five phialides. o Phialide releasing phialoconidia. p Phialoconidia with rounded or truncated ends, one distinctly longer, first formed phialoconidium 


\section{Teleomorph:}

Apothecia: Nail-shaped with a 1.1-1.4 (-2) mm long and $0.2-0.5 \mathrm{~mm}$ wide stipe and a disc diameter of $0.8-2.2(-4)$ $\mathrm{mm}$; arising from superficial pseudosclerotial plates on AMA; disc concave to convex, depending on the age, whitish to cream in color (Fig. 4b); surface of hymenium, stipe and receptacle smooth, stipe with a brown to black basal collar, containing a few irregularly shaped single crystals in crosssection. Asci clavate, distinctly long stipitate, $99-131 \mu \mathrm{m}(\varnothing$ $117.5, \pm 4.25)$ long, $7.5-8.5 \mu \mathrm{m}(\varnothing 8.2, \pm 0.18)$ wide at the widest point, eight-spored (Fig. 4i, j); apical pore blue in Lugol's reagent, Hymenoscyphus-type (Fig. 4d) (see Baral 1987), without croziers (Fig. 3e), ascospores biseriate (Fig. c), moderately "scutuloid," hyaline, non-septate, with two or more large (and some small) oil drops (living state in $\mathrm{H}_{2} \mathrm{O}$, Fig. 4f), 1-2 large confluent oil drops in LA (Fig. 4c), without setulae (Fig. 4f), 14-18.5 $\mu \mathrm{m}(\varnothing 15.98, \pm 0.51) \times 3.5-$ $4 \mu \mathrm{m}(\varnothing 3.83, \pm 0.11)$, becoming faintly brown and often one septate upon germination on AMA, forming one to two germ tubes (Fig. 4g), paraphyses cylindrical, hyaline, 2-3 $\mu \mathrm{m}$ thick, upper septum 37-64 $\mu \mathrm{m}$ below the apex, strongly refractive vacuolar bodies observed when mounted in $\mathrm{H}_{2} \mathrm{O}$ (Fig. 4h); ectal exipulum of textura prismatica with more or less rectangular cells, 24 $40 \times 4-10 \mu \mathrm{m}$, cortical cells hyaline (Fig. 4k), medullary excipulum hyaline, of textura intricata, outer part of textura porrecta, multi-guttulate in LA, cells $25-50 \times 3.7-$ $5.1 \mu \mathrm{m}$ (Fig. 41).

Anamorph (KUS-F52847_03): Phialophores arising on the superficial vegetative hyphae (Fig. 4n) or directly on pseudosclerotial plate on AMA, light brown, bearing 1-5 phialides; these with Chalara-like appearance (Fig. 4n, o), subcylindrical to obclavate, 16-22 $\mu \mathrm{m}$ long, venter inflated, short-cylindrical to ellipsoidal, mostly one, seldom two to three septate at the base, 8.5-13 $(\varnothing 10.45, \pm 1.00) \times 3.75-$ $4.75 \mu \mathrm{m}(\varnothing 4.35, \pm 0.20)$, collarette cylindrical 7.5-10.75 $(\varnothing$ $8.93, \pm 0.54) \times 2-2.75 \mu \mathrm{m}(\varnothing 2.28, \pm 0.17)$. Phialoconidia extruded in slimy droplets (Fig. 4o, p), one-celled, shortcylindrical to short-clavate, ends rounded or truncated, hyaline, mostly with one, seldomly two small oil droplets, $3-5$ ( $\varnothing$ $4.1, \pm 0.31) \times 2-2.5 \mu \mathrm{m}(\varnothing 2.05, \pm 0.07)$, the first formed phialoconidium being short-clavate, often with a truncated base, $7.5-12 \mu \mathrm{m}(\varnothing 8.85, \pm 0.99)$ (Fig. 4p). Germination of the phialoconidia was not observed.

Remarks: The teleomorph of $H$. occultus is morphologically very similar to the other species within the $H$. fraxineus group. The species differs from $H$. albidus and $H$. albidoides in the formation of an anamorph in culture. The anamorph revealed distinctly elongated and slightly longer phialoconidia (especially the first-formed conidium) compared to $H$. fraxineus and $H$. linearis. Another distinctive characteristic is the thinner and superficial pseudosclerotium on the petioles. In addition, it revealed the following unique nucleotide positions in the ITS sequence alignment, incorporating all reference strains of the $H$. fraxineus group (Online Resource 1): 53(C), 86(A), 94(T), 106(C), 331(C), 381(T), 384(A), 394(T), 397(T), 437(A), 453(T), 465-467(TCT) and 470(A). Further unique characteristics were revealed in the calmodulin alignment (Online Resource 2) at the following positions: 2(C), 14(G), 16(T), 34-38 (TTGAG), 57(C), 82(A), 99(C), 119(T), 158(T), 176(A), 181(A), 183(C), 235(A), 238(A), 241(T), 251-255 (deletion), $287(\mathrm{G})$ and 307(C).

\section{Discussion}

Our investigations of Korean collections confirmed the presence of $H$. fraxineus in Korea, as suggested by Han et al. (2014). In addition, two novel species of the $H$. fraxineus group were revealed. Both new species were recognized based on the GCPSR concept (Taylor et al. 2000), as well as on their morphology. The putative $H$. fraxineus sample KUSF52613_1 represented the only serious violation of genealogical concordance because, in marker EF1- $\alpha$, it grouped not with $H$. fraxineus samples but within the clade containing $H$. albidus. The same incongruence had already been detected and discussed in a phylogenetic study with Japanese samples of $H$. fraxineus by Zhao et al. (2012) and might represent a case of ancient hybridization or incomplete lineage sorting.

Even though our morphological analyses suggested that the two new species are theoretically distinguishable from other species of the $H$. fraxineus group, it will prove difficult to recognize them using solely morphological techniques, making them near-cryptic species. Cryptic specie complexes in fungi are frequently found (Grünig et al. 2004; e.g., see Barnes et al. 2004; Oono et al. 2014) because, in microbial organisms with a limited number of cells and morphological characteristics, it is assumed that genetic change occurs faster than morphological change (Taylor et al. 2006). However, cryptic species are often distinguishable based on their ecology or distribution range. In the current study, however, niche specialization is not evident at first glance. The three different species among the Korean collections occurred on the same host species, F. chinensis subsp. rhynchophylla, in the same host organ (petioles), and at roughly the same geographic location. Nevertheless, the collections with mature ascomata assigned to $H$. koreanus were consistently collected earlier in the year than those of $H$. fraxineus, indicating a slightly different fruiting time of these species in Korea. If this could be confirmed, it would be interesting to investigate why Korean $H$. fraxineus only fruits towards the end of summer, whereas the sporulation time in Europe can last from May to October, i.e., spanning almost the whole vegetation period (Gross et al. 2014a). The fruiting times of $H$. occultus and $H$. koreanus overlap since ascomata of both species were detected in the same collection (see Table 2, KUS-F52847_Fk1-3). The 
superficial pseudosclerotium of $H$. occultus further suggests the possibility of host tissue specialization. Organ specialization and different fruiting times seem to be common phenomena within the genus Hymenoscyphus; e.g., Hymenoscyphus fructigenus can be found on the woody part of the cone-like fruits of Alnus glutinosa in early Autumn, whereas Hymenoscyphus seminis-alni is only known from seeds (botanically = nut) of A. glutinosa, and apothecia are produced in late autumn (Baral 1996).

Homothallism and the ability to produce the anamorphic stage

Spontaneous ascocarp production in culture was observed for H. linearis, H. albidus, H. koreanus and H. occultus, suggesting these species are capable of self-fertilization and have a homothallic reproduction system (i.e., each haploid individual is sexually compatible with itself). Indeed, for $H$. albidus, the mating type locus was sequenced and a classical homothallic structure was identified (Gross et al. 2012). The fact that germination of ascospores was only observed after repeated sporulation might be due to the laboratory setup; e.g., unnatural light conditions.

Apparently, H. albidus does not produce any anamorphic stages in culture and it has been suggested this might be due to its homothallic reproduction system (Kirisits et al. 2013). In contrast, $H$. fraxineus develops a Chalara-like anamorph (see Kowalski 2006) and several lines of evidence indicate that phialoconidia of the Chalara anamorph serve as fertilizing units of the teleomorph of H. fraxineus (Gross et al. 2012). In our experiment, $H$. koreanus only developed phialides and did not produce any conidia. $H$. occultus frequently developed an anamorphic stage very similar to that of $H$. fraxineus (Kowalski 2006) and H. linearis (Gross et al. 2014, accepted article). It is possible that the optimal conditions for anamorph development of $H$. koreanus were not met. However, we believe that the observed structure represents a degenerated anamorphic stage because $H$. fraxineus and $H$. occultus developed a normal anamorph under the same conditions. From a pathological point of view, it would be interesting to investigate whether the anamorphs of $H$. occultus and $H$. linearis are functional since spermatia might enable interspecific hybridizations within the $H$. fraxineus group.

All the newly discovered members of the $H$. fraxineus group suggest that further members may be awaiting detection. The evolution of host specificity and the mating system of species within this group might have played a prominent role in speciation and deserve our future attention. The genus Fraxinus contains over 64 accepted taxa (Wallander 2008), evidencing the potential existence of as yet unknown species belonging to the $H$. fraxineus group. Those species currently detected certainly pose the risk of hybridizing with each other and becoming virulent or increasing in virulence, not only on F. excelsior in Europe, but also on Asian, North-American and other Fraxinus species in the world. Thus, intercontinental translocation and transference of these species should be avoided.

Acknowledgments We would like to thank O. Holdenrieder, T. Hosoya and V. Queloz for helpful discussions and suggestions; M. Berchtold, A. Duo and S. Stroheker for excellent technical assistance; H.O. Baral and an anonymous reviewer for constructive comments on a previous manuscript draft and C. Syrad for manuscript proofreading. We further acknowledge the Genetic Diversity Center of ETH Zurich for providing laboratory facilities. This study was supported by a grant from ETH Zurich (ETH-04 10-1).

Conflict of interest The authors declare that they have no conflict of interest.

\section{References}

Baral H-O, Queloz V, Hosoya T (2014) Hymenoscyphus fraxineus, the correct scientific name for the fungus causing ash dieback in europe. IMA Fungus 5(1):79-80. doi:10.5598/imafungus.2014.05.01.09

Baral HO (1987) Der Apikalapparat der Helotiales - Eine lichtmikroskopische Studie über Arten mit Amyloidring. Zeitschrift für Mykologie 53(1):119-135

Baral HO (1996) Hymenoscyphus seminis-alni, a new species of the H. fructigenus-complex. Mycotaxon 60:249-256

Baral HO, Bemmann M (2014) Hymenoscyphus fraxineus vs. Hymenoscyphus albidus - A comparative light microscopic study on the causal agent of European ash dieback and related foliicolous, stroma-forming species. Mycology 5(4):228-290. doi:10.1080/ 21501203.2014.963720

Barnes I, Crous PW, Wingfield BD, Wingfield MJ (2004) Multigene phylogenies reveal that red band needle blight of Pinus is caused by two distinct species of Dothistroma, D. septosporum and $D$. pini. Stud Mycol 50:551-565

Carbone I, Kohn LM (1999) A method for designing primer sets for speciation studies in filamentous ascomycetes. Mycologia 91(3): 553-556. doi:10.2307/3761358

Dlugosch KM, Parker IM (2008) Founding events in species invasions: genetic variation, adaptive evolution, and the role of multiple introductions. Mol Ecol 17(1):431-449. doi:10.1111/j.1365-294X.2007. 03538. $\mathrm{x}$

Drummond A, Rambaut A (2008) Tracer [Online]. Available at: http:// beast.bio.ed.ac.uk/Tracer. Accessed 25 October 2013

Gross A, Holdenrieder O (2013) On the longevity of Hymenoscyphus pseudoalbidus in petioles of Fraxinus excelsior. Forest Pathol 43(2):168-170. doi:10.1111/efp.12022

Gross A, Holdenrieder O, Pautasso M, Queloz V, Sieber TN (2014a) Hymenoscyphus pseudoalbidus, the causal agent of ash dieback. Mol Plant Pathol 15(1):109-117

Gross A, Hosoya T, Queloz V (2014b) Population structure of the invasive forest pathogen Hymenoscyphus pseudoalbidus. Mol Ecol 23(12):2943-2960. doi:10.1111/mec.12792

Gross A, Hosoya T, Zhao j, Baral HO (2014, accepted article) Hymenoscyphus linearis sp. nov. - Another close relative of the ash dieback pathogen $H$. fraxineus. Mycol Progr

Gross A, Zaffarano PL, Duo A, Grünig CR (2012) Reproductive mode and life cycle of the ash dieback pathogen Hymenoscyphus pseudoalbidus. Fungal Genet Biol 49(12):977-986. doi:10.1016/j. fgb.2012.08.008

Grünig CR, Linde CC, Sieber TN, Rogers SO (2003) Development of single-copy RFLP markers for population genetic studies of 
Phialocephala fortinii and closely related taxa. Mycol Res 107(11): 1332-1341

Grünig CR, McDonald BA, Sieber TN, Rogers SO, Holdenrieder O (2004) Evidence for subdivision of the root-endophyte Phialocephala fortinii into cryptic species and recombination within species. Fungal Genet Biol 41(7):676-687

Han J-G, Shrestha B, Hosoya T, Lee H-K, Sung G-H, Shin H-D (2014) First report of the ash dieback pathogen Hymenoscyphus fraxineus in Korea. Microbiology 42(4):391-396

Hosoya T, Otani Y, Furuya K (1993) Materials for the fungus flora of Japan (46). T Mycol Soc Jpn 34(4):429-432

Husson C, Scala B, Cael O, Frey P, Feau N, Ioos R, Marcais B (2011) Chalara fraxinea is an invasive pathogen in France. Eur J Plant Pathol 130(3):311-324. doi:10.1007/s10658-011-9755-9

Kirisits T, Dämpfle L, Kräutler K (2013) Hymenoscyphus albidus is not associated with an anamorphic stage and displays slower growth than Hymenoscyphus pseudoalbidus on agar media. Forest Pathol 43(5):386-389. doi:10.1111/efp. 12042

Kowalski T (2006) Chalara fraxinea sp. nov. associated with dieback of ash (Fraxinus excelsior) in Poland. Forest Pathol 36(4):264-270

Lanfear R, Calcott B, Ho SYW, Guindon S (2012) PartitionFinder: combined selection of partitioning schemes and substitution models for phylogenetic analyses. Mol Biol Evol 29(6):1695-1701. doi:10. 1093/molbev/mss020

Leach CM (1971) A practical guide to the effects of visible and ultraviolet light on fungi. In: Booth $\mathrm{C}$ (ed) Methods in microbiology, vol Volume 4. Academic, New York, pp 609-664. doi:10.1016/s05809517(09)70028-5

Marčiulynienè D, Cleary MR, Shabunin D, Stenlid J, Vasaitis R Detection of Hymenoscyphus pseudoalbidus in Primorye region, far east Russia; COST Action FP1103 Fraxback 4th MC meeting \& workshop "Frontiers in ash dieback research", 4-6th of September 2013, Malmö, Sweden; accessed online 21.01.2014 http://www. fraxback.eu/. 2013. p 42

McKinney LV, Thomsen IM, Kjær ED, Bengtsson SBK, Nielsen LR (2012) Rapid invasion by an aggressive pathogenic fungus (Hymenoscyphus pseudoalbidus) replaces a native decomposer (Hymenoscyphus albidus): a case of local cryptic extinction? Fungal Ecol 5(6):663-669. doi:10.1016/j.funeco.2012.05.004

Olson Å, Stenlid J (2002) Pathogenic fungal species hybrids infecting plants. Microb Infect 4(13):1353-1359
Oono R, Lutzoni F, Arnold AE, Kaye L, U’Ren JM, May G, Carbone I (2014) Genetic variation in horizontally transmitted fungal endophytes of pine needles reveals population structure in cryptic species. Am J Bot 101(8):1362-1374. doi:10.3732/ajb.1400141

Queloz V, Grünig CR, Berndt R, Kowalski T, Sieber TN, Holdenrieder O (2011) Cryptic speciation in Hymenoscyphus albidus. Forest Pathol 41(2):133-142. doi:10.1111/j.1439-0329.2010.00645.x

Ronquist F, Teslenko M, van der Mark P, Ayres DL, Darling A, Höhna S, Larget B, Liu L, Suchard MA, Huelsenbeck JP (2012) MrBayes 3.2: efficient Bayesian phylogenetic inference and model choice across a large model space. Syst Biol 61(3):539-542. doi:10.1093/sysbio/ sys029

Stamatakis A (2006) RAxML-VI-HPC: maximum likelihood-based phylogenetic analyses with thousands of taxa and mixed models. Bioinformatics 22(21):2688-2690. doi:10.1093/bioinformatics/ bt1446

Stergiopoulos I, Gordon TR (2014) Cryptic fungal infections: the hidden agenda of plant pathogens. Front Plant Sci 5:1-4

Taylor JW, Jacobson DJ, Kroken S, Kasuga T, Geiser DM, Hibbett DS, Fisher MC (2000) Phylogenetic species recognition and species concepts in fungi. Fungal Genet Biol 31(1):21-32. doi:10.1006/ fgbi.2000.1228

Taylor JW, Turner E, Townsend JP, Dettman JR, Jacobson D (2006) Eukaryotic microbes, species recognition and the geographic limits of species: examples from the kingdom Fungi. Philos T Roy Soc B 361(1475):1947-1963. doi:10.1098/rstb.2006.1923

Wallander E (2008) Systematics of Fraxinus (Oleaceae) and evolution of dioecy. Plant Syst Evol 273(1):25-49. doi:10.1007/s00606-0080005-3

White TJ, Bruns T, Lee S, Taylor J (1990) Amplification and direct sequencing of fungal ribosomal RNA genes for phylogenetics. In: Innis MA et al (eds) PCR protocols: A guide to methods and applications. Academic Press, Inc, San Diego and London, pp 315-322

Zhao Y-J, Hosoya T, Baral H-O, Hosaka K, Kakishima M (2012) Hymenoscyphus pseudoalbidus, the correct name for Lambertella albida reported from Japan. Mycotaxon 122:25-41. doi:10.5248/ 122.25

Zheng H-D, Zhuang W-Y (2013) Hymenoscyphus albidoides sp. nov. and H. pseudoalbidus from China. Mycol Progr 13(3):625-638. doi:10. 1007/s11557-013-0945-Z 